(c) American Dairy Science Association, 2004.

\title{
Cow-Level Prevalence of Paratuberculosis in Culled Dairy Cows in Atlantic Canada and Maine
}

\author{
S. L. B. McKenna, ${ }^{1}$ G. P. Keefe,${ }^{1}$ H. W. Barkema, ${ }^{1}$ J. McClure, ${ }^{1}$ \\ J. A. VanLeeuwen, ${ }^{1}$ P. Hanna, ${ }^{1}$ and D. C. Sockett ${ }^{2}$ \\ ${ }^{1}$ Atlantic Veterinary College, \\ University of Prince Edward Island, Charlottetown, Canada \\ ${ }^{2}$ Wisconsin Diagnostic Veterinary Laboratory, Madison 53705-4494
}

\section{ABSTRACT}

The prevalence of Mycobacterium avium ssp. paratuberculosis (Mptb) in culled dairy cattle in Eastern Canada and Maine was determined to be $16.1 \%$ (95\% confidence interval 13.8 to $18.3 \%$ ) based on a systematic random sample of abattoir cattle. Mesenteric lymph nodes and ileum from 984 cows were examined by histologic and bacteriologic methods. Histological testing was far less sensitive than bacteriologic methods for detecting infected cattle. A seasonal pattern of positive cows was also detected, with the highest proportion of cows being $M p t b$-positive in June (42.5\%). Overall, body condition score was not associated with prevalence of $M p t b$ isolation.

(Key words: paratuberculosis, prevalence, culture, slaughterhouse)

\begin{abstract}
Abbreviation key: HEY = Herrold's egg yolk, $\boldsymbol{M p t b}=$ Mycobacterium avium ssp. Paratuberculosis.
\end{abstract}

\section{INTRODUCTION}

Mycobacterium avium ssp. paratuberculosis (Mptb) is the bacterium that causes Johne's disease, a chronic wasting disease of ruminants associated with infection of the animal's gastrointestinal tract. The effect this infection has on health and productivity has been studied; one estimate of cost for Johne's positive herds that had $10 \%$ of cull cows with clinical signs was over US $\$ 200$ per cow (Ott et al., 1999). In another study within the region this study was performed, using serologic data and partial-budget modeling the annual cost for an average, infected, 50 cow herd was CDN\$2472 (Chi et al., 2002). Although strategies exist in many

Received October 22, 2003.

Accepted April 8, 2004.

Corresponding author: S. L. B. McKenna: e-mail: slmckenna@ upei.ca. places to control this disease, it still continues to affect the health and productivity of cattle worldwide.

Before a control and prevention program for Johne's diseases can be designed, both herd and cow-level prevalence of $M p t b$-infection should be known. Several regional and national surveys have been conducted to estimate the prevalence of $M p t b$-infection. However, different methods were used to estimate the prevalence of $M p t b$-infection including: 1) serological testing; 2) bacteriological culture of fecal samples; 3 ) bacteriological culture of tissue samples from slaughterhouses; and 4) PCR on tissue, blood, or bulk milk. Moreover, sampling strategies differed among the studies. Thus, it is difficult to directly compare the results of different studies. During the early 1990s, the herd-level prevalence of Johne's disease in countries with a significant dairy industry had been calculated to be approximately 10\% (Sockett, 1996). However, recently, in Belgium (Boelaert et al., 2000), The Netherlands (Muskens et al., 2000), Denmark (Nielsen et al., 2000), Canada (VanLeeuwen et al., 2001), and the United States (Wells and Wagner, 2000) herd-level prevalence has been estimated to be 30 to $50 \%$, based on an observed increased frequency of clinical disease, occurrence of Johne's disease in areas where the disease was formerly unknown, and serological prevalence studies. Due to the relatively low sensitivity of serological tests, they are more accurate at determining herd-level rather than cow-level prevalence. Serologic studies performed to determine cow-level prevalence have indicated a prevalence between 1.2 and 8.8\% (Boelaert et al., 2000; Jakobsen et al., 2000; Muskens et al., 2000).

In comparing different studies involving prevalence of disease, part of the difficulties arise from variation in the definition of what constitutes a positive case. In some cases, positive serologic result is the case definition and in others, such as this study, a positive result is presence of bacteria. A previous study done in Atlantic Canada using serologic testing of dairy cows reported a cow-level prevalence of $2.6 \%$, with an estimated true 
herd-level prevalence of $30 \%$ (VanLeeuwen et al., 2001). This study used ELISA for classification of cow status on a stratified (cows, farms) random sample. The ELISA test was assumed to have a sensitivity of $45.5 \%$ (Collins and Sockett, 1993), but more recent work has estimated the sensitivity to be closer to $15.4 \%$ in low fecal shedders ( 1 to $10 \mathrm{cfu}$ to $88.1 \%$ in high fecal shedders (>100 cfu) (Dargatz et al., 2001).

Most estimates of ELISA performance are based on culture methods as the comparison gold standard (most often being fecal culture). The relationship between fecal culture sensitivity and ELISA sensitivity depends highly on the stage of the disease in an individual animal. In the earlier stages of disease, there is a lower sensitivity for both methods (Whitlock et al., 2000). However, tissue culture in comparison with repeated fecal culture reveals that animals that are fecal culture negative may actually be tissue culture positive, therefore resulting in a higher sensitivity for tissue culture (Pavlik et al., 2000).

Body condition scores of cattle are believed to be linked to the prevalence of $M p t b$ because clinical Johne's disease results in an emaciated, debilitated cow with diarrhea. This has been demonstrated in Australian sheep. Animals with low BCS $(<2$ out of 5$)$ had a $M p t b$-prevalence of $51.5 \%$ compared with the sheep with a higher BCS (2 to 4 out of 5 ), where the prevalence was only $19.3 \%$ (Hope et al., 2000). Additionally, BCS in sheep has an affect on serologic test sensitivity. In evaluating test sensitivity in sheep, animals with lower $\mathrm{BCS}(\leq 2)$ resulted in test sensitivity of 53\%, and sheep with a BCS $\geq 3$ resulted in a calculated test sensitivity of $16 \%$ (Sergeant et al., 2003). Therefore, interpreting BCS and prevalence becomes difficult due to the effects of changes in sensitivity. The apparent link of BCS and prevalence could be due to its effects on sensitivity or an association between BCS and stage of infection. In either case, an association between BCS and infections could be used to select cows for laboratory diagnosis of Mptb. No data on the association between Mptbinfection and BCS in the bovine are currently available.

The goals of this study were 1) to estimate slaughtered cow-level prevalence of $M p t b$ in Atlantic Canada and the US state, Maine and 2) to study the association between BCS and prevalence of infection with $M p t b$. Testing for Mptb usually consists of serology (ELISA), culture (fecal or tissue), or use of DNA detection methods such as PCR techniques. In this study, a culture of infected tissues was used to determine infection with $M p t b$ because its sensitivity may be greater than that of a fecal culture, as some repeatedly fecal-culture negative animals will be tissue positive (Pavlik et al., 2000). However, this may be influenced by the stage of disease. Because culture of tissue is impractical to perform on a large number of live animals, $M p t b$-prevalence was estimated in slaughterhouse cows.

\section{MATERIALS AND METHODS}

\section{Sample Population}

For the period from January to October 2001, excluding April and May, during which a labor dispute at the slaughterhouse halted collection of samples, a weekly visit was made to a local slaughterhouse for the dairy cow-processing day. The slaughterhouse was located in Moncton, New Brunswick, and slaughtered cows were primarily from the 4 Atlantic Canadian Provinces: New Brunswick, Nova Scotia, Prince Edward Island, and Newfoundland. Additionally, this slaughterhouse occasionally processed animals purchased from buyers located in the US state, Maine. These animals were identified as American cattle for accurate data analysis.

The sample size required was estimated using seroprevalence data from a previous study done in the area, which resulted in a cow-level apparent prevalence of $2.6 \%$ (VanLeeuwen et al., 2001), with a 95\% level of confidence and a desired accuracy of the prevalence to be within $1 \%$ of the true value. This yielded a number of 973 cows needed for the study (Win Episcope 2.0). Animals that were not of dairy breeds were not included in the study. In addition, to ensure that cows were all at least 20 mo old, animals that did not appear to have udder development consistent with previous lactation were excluded. Sampling was conducted using a systematic random sample, in which every third slaughtered cow was selected. Slaughterhouse staff were the only individuals who had any control over the order and grouping of cows that entered the production line. Using this methodology, 984 cows were sampled over the 10 -mo period. Holstein-Friesian was the predominant breed, making up 961 of the 984 cows.

\section{Sample Collection}

Three people were involved with sample collection. As animals entered the kill line, the first person recorded the cow's BCS on a 5-point scale with one-quarter point divisions (Ferguson et al., 1994). The same individual recorded breed and BCS on every visit to the slaughterhouse in an attempt to avoid interperson variation between visits. For consistency, all assessments of body condition were performed immediately after the cow was stunned and hanged, because this was often the first time the scorer would see the animal.

Upon exsanguination, a free catch blood sample of approximately $80 \mathrm{~mL}$ was obtained. The animal was then identified with numbered colored tags to track the carcass to the evisceration area. At the evisceration 
table, a second person identified the gastrointestinal tract of each study animal with a separate tag containing a unique numeric identifier. At a subsequent station, a third person collected at least 2 lymph nodes in the mesenteric chain in the region of the ileum, with focused efforts to retrieve the lymph node in the ileocecal region, similar to methods described by Benedictus and Bosma (1985). Additionally, a 5- to 10-cm segment of terminal ileum was removed approximately $25 \mathrm{~cm}$ proximal to the ileo-cecal junction. Samples were placed in individual containers labeled with the numeric identifier for each animal and transported fresh in a cooler with ice packs to the Atlantic Veterinary College.

After returning from the slaughterhouse, samples were processed for storage within $8 \mathrm{~h}$ of collection. From each sample of ileum and lymph node, a section was preserved in $10 \%$ neutral buffered formalin, and the remainder was frozen at $-80^{\circ} \mathrm{C}$. Physical characteristics of the samples were recorded, indicating whether there was gross thickening of the ileum, loose feces, or enlarged lymph nodes.

\section{Histological Preparation}

After formalin fixation, samples were trimmed and embedded in paraffin wax. They were subsequently sectioned and Ziehl-Neelsen staining was performed (Murray, 1999). All histopathological examinations were performed by a board-certified pathologist (American College of Veterinary Pathology) at the Atlantic Veterinary College Diagnostic Services (Charlottetown, PEI, Canada). The examination was to search for lesions similar to those described by Buergelt et al. (1978).

\section{Culture Technique}

For each cow, the collected lymph nodes were pooled together to comprise one culture sample, and the ileum was identically processed for a second cultured sample. Tissue sample processing for culture was done using a protocol developed by researchers in Pennsylvania (Wells et al., 2002). Briefly, samples were thawed and a 2-g piece of sample was weighed out on an individual disposable weigh boat to decrease the risk of crosscontamination. Each sample was then placed in a Tekmar bag along with $25 \mathrm{~mL}$ of $0.75 \%$ hexadecylpyridinium chloride solution for decontamination. The sample was placed in a stomacher for a minimum of $1 \mathrm{~min}$ to pulverize the sample. Subsequently, each sample was left undisturbed for 30 min to allow fat and large pieces of tissue to separate out while being bathed in the hexadecylpyridinium chloride decontamination solution. A $10-\mathrm{mL}$ sample of the pulverized fluid was transferred into another sterile tube containing a further $10 \mathrm{~mL}$ of hexadecylpyridinium chloride solution, used for the second decontamination stage in the procedure. After a minimum of $3 \mathrm{~h}$ in the second decontamination stage, samples were centrifuged at $900 \times g$ for $30 \mathrm{~min}$. The supernatant was discarded, and the pellet was resuspended with a combined half strength brain heart infusion broth that contained $0.1 \%$ nalidixic acid, $0.1 \%$ vancomycin, and $0.05 \%$ amphotericin $B$. This mixture was vortexed and placed into sterile cryogenic tubes to be incubated for 12 to $14 \mathrm{~h}$ at $37^{\circ} \mathrm{C}$ to allow for $M p t b$ growth and to allow an opportunity for the antimicrobials to more effectively decrease levels of background contaminants. After the incubation phase, the tubes were slowly cooled and then refrozen at $-80^{\circ} \mathrm{C}$.

The frozen, processed culture mixture was sent to the Wisconsin Veterinary Diagnostic Laboratory for culturing. At the Wisconsin laboratory, samples were thawed, and $1 \mathrm{~mL}$ from each of the 1968 tubes was inoculated into a VersaTREK broth solution media bottle (TREK Diagnostic Systems, Cleveland, OH), supplemented with egg yolk, antibiotics $(0.1 \%$ nalidixic acid, $0.1 \%$ vancomycin, and $0.05 \%$ amphotericin $\mathrm{B}$ ), and $\mathrm{My}$ cobactin J (Allied Monitor Inc., Fayette, MO). The inoculated media were incubated for $6 \mathrm{wk}$ at $37^{\circ} \mathrm{C}$. After 6 wk of incubation, each bottle was examined for acid-fast bacteria. For this process, each bottle was vigorously shaken for a minimum of $60 \mathrm{~s}$ using a Mistral multimixer (Laboratory Line Instruments Inc., Melrose Park, IL). A sample (1 drop) from each bottle was placed on a microscope slide, with a total of 3 samples per slide. The slides were air-dried, then heat fixed, and an acid-fast staining process was performed on each slide (Murray, 1999). Each slide was then examined at oilimmersion $(100 \times)$ for the presence of acid-fast bacteria.

Every sample that was positive or suspicious for acidfast bacteria was subcultured onto 2 slants of Herrold's egg yolk (HEY) media (1 tube containing mycobactin and 1 tube without mycobactin). The HEY tubes were examined weekly for $6 \mathrm{wk}$. Isolates that grew well (10 colonies or more) on tubes that contained mycobactin (mycobactin dependent) and had minimal or no growth on the tubes that did not contain mycobactin were identified as Mptb. Isolates that grew well on both HEY slants or had fewer than 10 colonies on the slant containing mycobactin were tested by the PCR.

\section{PCR Technique}

For DNA extraction, lysis by the boiling method was used. Briefly, 1 or 2 bacterial colonies from each HEY slant were suspended in $100 \mu \mathrm{L}$ of lysis buffer containing HPLC grade water with $1 \%$ Triton-X-100 (Fisher Scientific, Pittsburgh, PA), $1 \mathrm{~m} M$ EDTA, and 
Table 1. Prevalence of infection with Mycobacterium avium ssp. paratuberculosis in 832 Atlantic Canadian and 152 Maine (United States) cull dairy cows by sample site.

\begin{tabular}{llclr}
\hline & Ileum & Lymph nodes & Both & \multicolumn{1}{l}{ Total } \\
\hline Atlantic Canada & $67(8.1 \%)$ & $83(10.0 \%)$ & $25(3.0 \%)$ & $125(15.1 \%)$ \\
Maine & $18(11.8 \%)$ & $27(17.8 \%)$ & $10(6.6 \%)$ & $33(21.7 \%)$ \\
Total & $85(8.5 \%)$ & $110(11.1 \%)$ & $35(3.5 \%)$ & $158(16.1 \%)$ \\
\hline
\end{tabular}

$10 \mathrm{~m} M$ Tris- $\mathrm{HCl}(\mathrm{pH}$ 8.0). This mixture was placed in a heat block (Fisher Scientific) and heated to $100^{\circ} \mathrm{C}$ for 30 min to lyse cells. Following centrifugation for 2 min at $13,000 \times g$ in a microcentrifuge, the cell-free supernatant containing DNA was transferred to a fresh tube and used for PCR.

The oligonucleotide primers used for the IS900 and F57 genetic elements have previously been described by Vary et al. (1990) and Poupart et al. (1993). The forward primer designated IS900/ $150 \mathrm{C}$ and the reverse primer designated IS900/921 were used for IS900; the forward primer designated F57a and the reverse primer designated F57b were used for F57. The primer pair for IS900 results in the amplification of a 229-bp fragment, and the primer pair for F57 results in the amplification of 439-bp fragment, respectively.

Amplification reactions were performed in a total volume of $50 \mu \mathrm{L}$ containing $10 \mathrm{~m} M$ Tris- $\mathrm{HCl}(\mathrm{pH}$ 8.3); 1.5 $\mathrm{m} M \mathrm{MgCl}_{2} ; 50 \mathrm{~m} M \mathrm{KCl} ; 0.001 \%$ gelatin; $200 \mu M$ of each deoxynucleoside triphosphate (dATP, dCTP, dGTP, dTTP); $1 \mu M$ of each primer; $1.25 \mathrm{U}$ of Amplitaq (Applied Biosystems, Foster City, CA); and $5 \mu \mathrm{L}$ of the boiled cell lysate for the monoplex PCR. The PCR assay was carried out in a Perkin-Elmer 2400 thermocycler (Perkin Elmer Corp., Norwalk, CT) comprising 5 min of preincubation at $94^{\circ} \mathrm{C}$, followed by 35 cycles of $1 \mathrm{~min}$ at $94^{\circ} \mathrm{C}, 1 \mathrm{~min}$ at $54^{\circ} \mathrm{C}$, and $1 \mathrm{~min}$ at $72^{\circ} \mathrm{C}$. Final extension was performed for $7 \mathrm{~min}$ at $72^{\circ} \mathrm{C}$. A single colony from a HEY culture positive slant was used as the DNA positive control. The negative control was a reaction mixture containing all reagents but no DNA template. The PCR products were visualized by electrophoresis on a $1 \%$ agarose gel following standard procedures. Bacterial isolates positive to both the F57 and IS900 genetic elements were classified as positive for $M p t b$.

\section{Statistical Analysis}

Prior to statistical analyses, observations were checked for unlikely values; no data were excluded for this reason. Missing values (11 of the 984 cows did not have a BCS recorded) routinely caused a record to be excluded if the analysis included BCS. The distribution of BCS was testing for normality using the AndersonDarling Normality Test. Mean BCS of Canadian and US cows was tested with Student's $t$-test. Unconditional associations between prevalence of infection with both month and category of BCS (divided into 4 categories: $<2,2$ to 3,3 to 4 , and $>4$ ) were evaluated using a $\chi^{2}$ on a contingency table. A logistic regression was performed using the $M p t b$ culture result as a dependent variable and BCS and place of origin as independent variables, with BCS categorized in the same 4 categories to avoid assumptions of linearity. Statistical significance was defined at $P=0.05$. All data analysis was done using Stata 7.0 (Stata Corporation, College Station, TX).

\section{RESULTS}

Overall prevalence of infection with $M p t b$ was $16.1 \%$ (95\% CI: 13.8 to $18.3 \%$ ). In total, $8.5 \%$ of the cows had positive ileum cultures and $11.1 \%$ of the cows had positive lymph node cultures. There were 37 cultures that had positive slants without mycobactin that were confirmed positive by both IS900 and F57 PCR analysis. Only $3.5 \%$ of the cows were $M p t b$-positive at both sites (Table 1). Prevalence of $M p t b$ in the 832 Atlantic Canadian cows was $15.1 \%$ (95\% CI: 12.7 to $17.5 \%$ ). Prevalence of infection in the 152 cows originating from the US state, Maine was 21.7\% (95\% CI: 15.2 to $28.3 \%$ ). The odds of a US cow being positive were $1.56(P=$ 0.04 ) times greater than a cow from Canada, when controlled for BCS. There was no detected interaction between BCS and country of origin.

Analysis of the relationship between the physical characteristics of samples (gross thickening of the ileum, loose feces, or enlarged mesenteric lymph nodes) revealed that of the 984 cows, only 134 cows had physical attributes in one of these 3 categories. Of those 134 cows, only 27 (20.1\%) cows were actually culturepositive and only $1(0.7 \%)$ was histologically positive (Table 2). All 7 cows that were histologically indicative of Johne's disease were also Mptb-positive (Table 2).

The monthly proportion of $M p t b$-positive cows varied from 2.4 to $42.5 \%$, with $82.4 \%$ of the positive cows identified during 3 of the 8 mo the samples were collected from the plant. The highest proportion of culture-positive cows was identified in February, March, and June (Figure $1 ; \chi^{2}=141.2,7 \mathrm{df}, P=0.001$ ). In June, prevalence of $M p t b$-positive cows was the highest at $42.5 \%$. In all months but June, proportion of $M p t b$-isolation 
Table 2. Distribution of observed physical characteristics and pathological changes among Mycobacterium avium ssp. paratuberculosis-positive (Mptb-positive) cull dairy cattle.

\begin{tabular}{llll}
\hline & $\begin{array}{l}\text { Total } \\
\text { Mptb-positive } \\
(\mathrm{n}=158)\end{array}$ & $\begin{array}{l}\text { Atlantic Canada } \\
\text { Mptb-positive } \\
(\mathrm{n}=125)\end{array}$ & $\begin{array}{l}\text { Maine } \\
\text { Mptb-positive } \\
(\mathrm{n}=33)\end{array}$ \\
Observations in 984 cull dairy cows & 23 & 18 & 5 \\
Macroscopic thickened ileum $(\mathrm{n}=112)$ & 7 & 6 & 1 \\
Histological changes $(\mathrm{n}=7)$ & 5 & 4 & 1 \\
Loose feces $(\mathrm{n}=29)$ & 1 & 1 & 0 \\
Enlarged lymph nodes $(\mathrm{n}=18)$ & &
\end{tabular}

from lymph nodes was higher than from the ileum $\left(\chi^{2}=\right.$ $52.0,1 \mathrm{df}, P=0.001)$. In June, $34.1 \%$ of the ileum samples were $M p t b$-positive, whereas only $21.2 \%$ of the lymph nodes were culture-positive (Figure 2).

\section{Body Condition Score}

The BCS for all cows in this study was normally distributed (Anderson-Darling Normality Test, $P<$ 0.001). Therefore, although BCS is an ordinal scale, using averages is valid. Of the culture-positive cows, $73.0 \%$ had a BCS of $\geq 2.75$, which is a relatively good conditioned dairy cow. Average BCS was 3.0 (95\% CI: 3.0 to 3.1) for the $M p t b$-positive Canadian cows and 2.8 (95\% CI: 2.7 to 2.9 ) for the $M p t b$-positive cows from Maine. The range of BCS for US cows was 1.5 to 4.25 , with quartiles of $\mathrm{Q} 1=2.25$, median $=2.75$, and $\mathrm{Q} 3=$ 3.25. The range of BCS for Canadian cows was 1.25 to 4.5 , with quartiles of $\mathrm{Q} 1=2.75$, median $=3.0$, and $\mathrm{Q} 3=3.5$.

Overall, average BCS was higher in the Canadian cows than in cows from Maine $(P=0.0003)$. Prevalence of $M p t b$-infection was not associated with low BCS (Fig-

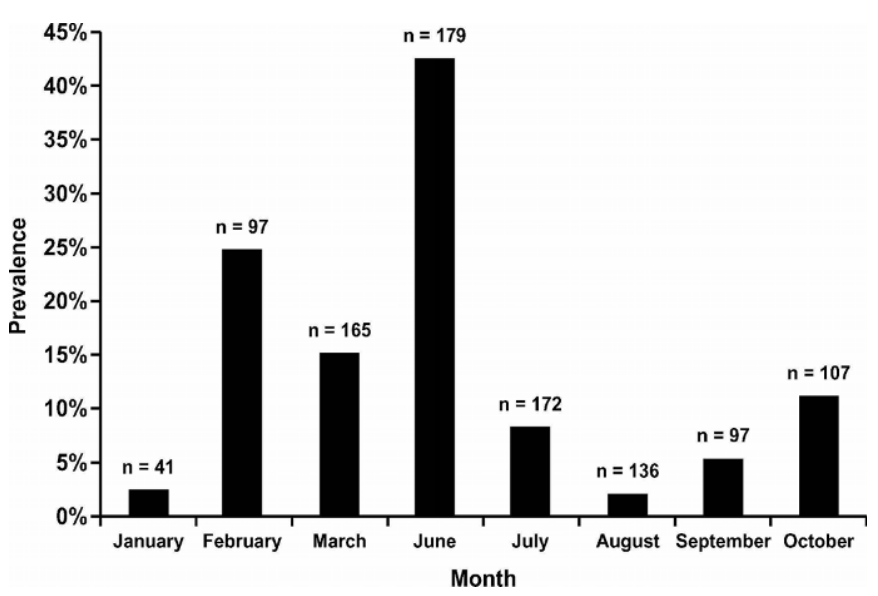

Figure 1. Prevalence of Mycobacterium avium ssp. paratuberculosis in 984 cull dairy cattle by month sampled. ure $\left.3 ; \chi^{2} P=0.33\right)$. Ileum was the more prevalent $M p t b$ positive site at lower BCS with a prevalence of $16.0 \%$ for cows with body condition of $\leq 2.5$ compared with the prevalence of $8.9 \%$ for lymph nodes (Figure 4 ), but this was not statistically significant $(P=0.18)$.

\section{DISCUSSION}

The prevalence of $M p t b$ found in this study of $16.1 \%$ is substantially higher than a previous estimated seroprevalence of $2.6 \%$ of randomly sampled cows from a serological study done $5 \mathrm{yr}$ ago of the Atlantic provinces (VanLeeuwen et al., 2001). It is unlikely that we would see such a dramatic rise in prevalence in a 4-yr period when dealing with a slow growing pathogen. These comparisons are apparent prevalences, not true prevalences. Likely, much of the difference in prevalence estimates between the 2 studies is due to an underestimation of the true prevalence based upon the low diagnostic sensitivity of the ELISA used by VanLeeuwen et al. (2001). Culture of tissue is expected to be more sensitive, because of detection of earlier stages of infection than an ELISA, therefore the apparent prevalence will be closer to the true prevalence.

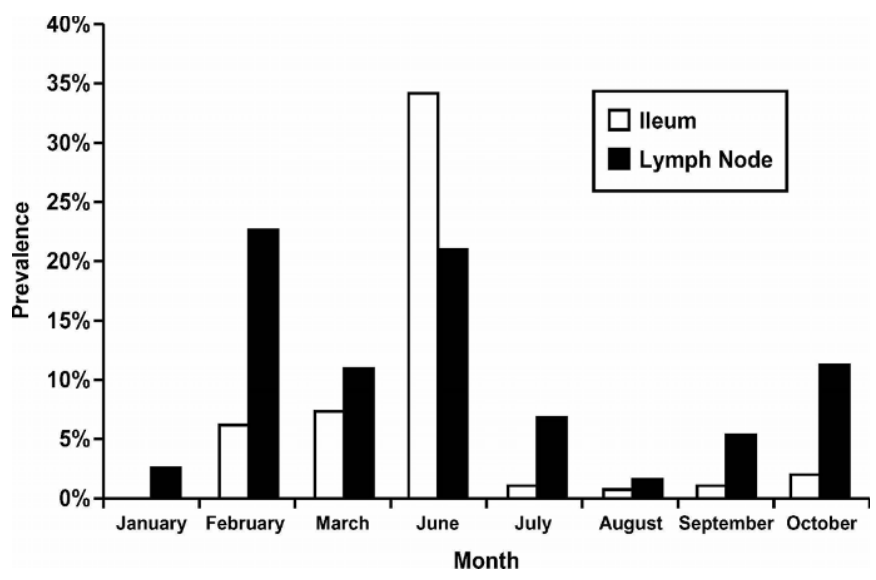

Figure 2. Prevalence of isolation of Mycobacterium avium ssp. paratuberculosis in the 2 sampled tissue sites, ileum and mesenterial lymph nodes, by month sampled. 


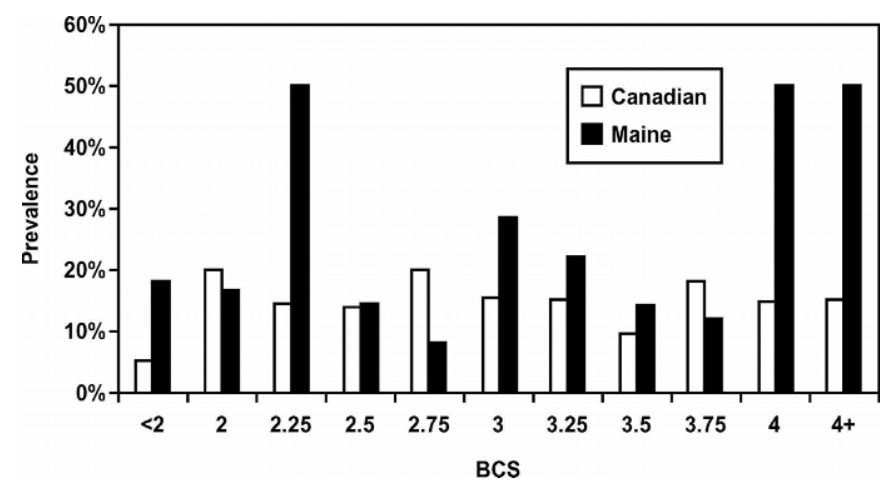

Figure 3. Distribution of BCS and prevalence of infection with Mycobacterium avium ssp. paratuberculosis for Canadian and Maine cows.

The process of thawing and refreezing the samples may have caused the loss of viable organisms. The full magnitude of this loss is not known, but using data from losses accrued during freezing fecal samples, the number of organisms may be reduced by one-tenth (Richards, 1981). Conclusions from this study were that it would not change the status of high and moderate shedders, but low shedders may appear as negative cows after 2 freeze-thaw cycles. The impact of this unavoidable need to freeze samples is that there may be cows in this study that are infected with low numbers of $M p t b$ that resulted in negative cultures. This would result in biasing our prevalence estimate to be lower than what it truly was.

For some diseases, study of cows sampled at a slaughterhouse may not be representative of the general population. However, for $M p t b$, the estimate of the prevalence of infection derived from this sample is likely to be a very good estimate of the prevalence in the population of dairy cows for the following reasons. First, the slaughterhouse used in the study was one of only 2 federally inspected plants in the region. The other

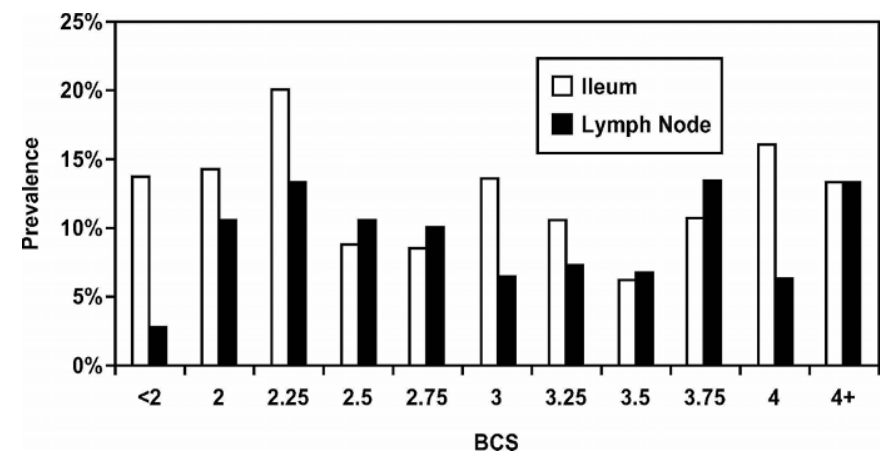

Figure 4. Distribution of BCS and Mycobacterium avium ssp. paratuberculosis prevalence for 2 different tissue sites. plant had a policy of not slaughtering dairy animals. As a result, the great majority of culled dairy cows from the region are killed at the study facility. Second, most $M p t b$-infections occur early in life (Larsen et al., 1975) and are presumably persistent throughout life since studies of repeated ELISA and fecal testing fail to indicate cows that self-cure (van Schaik et al., 2003). As a result, the actual prevalence of infection within a birth cohort will remain relatively constant over life. Using less sensitive diagnostic methods (ELISA, fecal culture), which more readily identify late stage disease, may suggest a higher prevalence in certain age groups. Using more sensitive techniques, and limiting the population under study to animals that had calved at least once ( $>2$ yr of age), allows the study to closely approximate the true disease prevalence. Finally, Johne's disease appears to have relatively little effect in terms of causing premature culling (Tiwari et al., 2003). However, even if it did cause premature culling, the estimate of the prevalence of infection would only be affected if the incidence of new infections was rapidly increasing (or decreasing) across birth cohorts. In the absence of a specific control program in the region, it is unlikely that the incidence is rapidly decreasing, and there is no reason to believe the region is experiencing a rapid rise in the incidence of new infections. Consequently, the estimate of the prevalence derived from this slaughterhouse study is likely to be a good estimate of the prevalence of infection in the dairy cow population in the region.

Unfortunately, there was no cattle identification and registration system in place at the time of data collection that would have facilitated tracing each infected animal to its herd of origin. For this reason, no estimate of herd-level prevalence could be made. Such information might have proven to be quite valuable for future monitoring of disease distribution and potentially identifying concentrations of diseased animals within highly infected herds.

Historically, the isolation of a slow growing acid-fast positive bacillus from a cow that required exogenous mycobactin for growth was sufficient to identify a culture isolate as Mptb (Chiodini et al., 1984). However, in this study, 37 of the 158 culture positives (23.4\%) failed to demonstrate mycobactin dependence but were positive based on 2 PCR tests. This was probably due to intracellular stores of mycobactin from the TREK media that enabled growth of the organism on HEY slants that did not contain mycobactin. This hypothesis was later validated by subculturing acid fast colonies from the 37 questionable HEY tubes without mycobactin onto HEY slants with and without mycobactin and demonstrating subsequent mycobactin dependence. This observation demonstrated that PCR was superior 
to subculturing onto HEY slants for identifying $M p t b$ from acid-fast positive broth media cultures.

Because reports by Englund et al. (2002) and Cousins et al. (1999) suggest that IS900 may not be $100 \%$ specific for $M p t b$, we elected to also test the questionable samples for the F57 gene product, as well as the IS900. F57 has been shown to be $100 \%$ specific for Mptb (Poupart et al., 1993; Coetsier et al., 2000). All 37 positive IS900 samples were also positive for F57.

Common dogma among farmers with respect to $M p t b$ is that infected cows are more likely to be thin and in poor body condition. Animals infected with $M p t b$ that are in the latter stages of disease are expected to be in poor body condition due to the onset of clinical signs, but that change may be gradual (Whitlock, 1996). Although early disease stage animals would not be expected to have dramatic weight loss, on the continuum of disease, from early to later stages represented in this large sample, some association between BCS and infection status could be anticipated. In our study, there was no association between BCS and infection status, with the majority of the culture-positive cows being in good condition. In fact, $73 \%$ of positive cows had a BCS of $\geq 2.75$, which is a favorable score for a lactating Holstein-Friesian cow. However, we do not have data on the stage of lactation of these cows. Unfortunately, there also is no available data to confirm whether these animals were fecal shedders, which might indicate the stage of infection. Due to the fact that only 7 of the 160 culture positive animals had histological evidence of infection, the argument could be made that the other 153 animals were in an early stage of the disease and had not reached a level of infection that would result in poor condition. Based upon findings from this study, BCS at slaughter is not a valid method to help find cows with $M p t b$. This study confirms that although cows may seem to be healthy and in good body condition, they may be carrying $M p t b$ and are undetectable as affected cows.

The finding of higher prevalence within the spring season was unexpected and difficult to explain. Cetinkaya et al. (1996) found a similar trend in the spring compared with the fall, but the difference was not significant. A high proportion of positive samples were collected in June, with a cluster of $M p t b$-positive cows on the third sampling day in June. It could be possible that a herd with a high prevalence of Johne's had just gone through a dispersal and an extraordinary number of positive animals arrived at slaughter the same day. It is unlikely that this high number of positives on a single day was a contamination issue because the same procedure was used throughout sampling. Also, sample preparation was done on 2 different occasions so that samples of ileums and lymph nodes were never handled on the same day. No $1 \mathrm{~d}$ of sample processing had a disproportionately high number of positives.

Peculiarly, in every month, a higher proportion of lymph nodes than ileum were $M p t b$-positive, except for the month of June, where the proportion of ileum-positive samples peaked. The higher proportion of $M p t b$ positive ileum samples in June could be due to cows being on pasture for approximately $1 \mathrm{mo}$ (a common practice in Atlantic Canada) and being exposed to higher numbers of $M p t b$ microbes and a possible pass through the gastro-intestinal tract effect. This may be due to cow exposure or simple increased stress during changing of feeding practices and environment, which could induce progress of the disease and shedding of $M p t b$. Although it is impossible to determine whether this is plausible from this study, further investigation may be warranted to better describe this seasonal finding.

The high prevalence of $M p t b$-infection in slaughter cows found in this study illustrates the need for Johne's disease control program initiatives to be established within this region. Other regions would likely find a similar prevalence if a tissue culture strategy were used.

\section{ACKNOWLEDGMENTS}

The authors would like to thank Theresa Rogers, Lloyd Dalziel, Rick Milton, the AVC Farm Service summer students, and the staff of the Wisconsin Veterinary Diagnostic Laboratory for assistance in making this project a reality.

\section{REFERENCES}

Benedictus, G., and J. Bosma. 1985. Paratuberculosis: A surgical method of diagnosis in practice. Vet. Q. 7:217-221.

Boelaert, F., K. Walravens, P. Biront, J. P. Vermeersch, D. Berkvens, and J. Godfroid. 2000. Prevalence of paratuberculosis (Johne's disease) in the Belgian cattle population. Vet. Microbiol. 20:269-281.

Buergelt, C. D., C. Hall, K. McEntee, and J. R. Duncan. 1978. Pathological evaluation of paratuberculosis in naturally infected cattle. Vet. Pathol. 15:196-207.

Cetinkaya, B., K. Egan, D. A. Harbour, and K. L. Morgan. 1996. An abattoir-based study of the prevalence of subclinical Johne's disease in adult cattle in southwest England. Epidemiol. Infect. 116:373-379.

Chi J., J. A. VanLeeuwen, A. Weersink, and G. P. Keefe. 2002. Direct production losses and treatment costs from bovine viral diarrhoea virus, bovine leukosis virus, Mycobacterium avium subspecies paratuberculosis, and Neospora caninum. Prev. Vet. Med. 55:137-153.

Chiodini, R. J., and H. J. van Kruiningen. 1986. The prevalence of paratuberculosis in culled New England cattle. Cornell Vet. 76:91-104.

Chiodini, R. J., J. J. Van Kruiningen, and R. S. Merkal. 1984. Ruminant paratuberculosis (Johne's disease): The current status and future prospects. Cornell Vet. 74:217-262.

Coetsier, C., P. Vannuffel, N. Blondeel, J. F. Denef, C. Cocito, and J. L. Gala. 2000. Duplex PCR for differential identification of 
Mycobacterium bovis, $M$. avium, and M. avium subsp. paratuberculosis in formalin-fixed paraffin-embedded tissues from cattle. J. Clin. Microbiol. 38:3048-3054.

Collins, M. T., and D. C. Sockett. 1993. Accuracy and economics of the USDA-licensed enzyme-linked immunosorbent assay for bovine paratuberculosis. JAVMA 203:1456-1463.

Cousins, D. V., R. Whittington, I. Marsh, A. Masters, R. J. Evans, and P. Kluver. 1999. Mycobacteria distinct from Mycobacterium avium subsp. paratuberculosis isolated from faeces of ruminants possess IS900-like sequences detectable by IS900 polymerase chain reaction implications for diagnosis. Mol. Cell. Probes 14:431-442.

Dargatz, D. A., B. A. Byrum, L. K. Barber, R. W. Sweeney, R. H. Whitlock, W. P. Shulaw, R. H. Jacobson, and J. R. Stabel. 2001. Evaluation of a commercial ELISA for diagnosis of paratuberculosis in cattle. JAVMA 218:1163-1166.

Englund, S., G. Bölske, and K. E. Johansson. 2002. An IS900-like sequence found in a Mycobacterium sp. other than Mycobacterium avium subsp. paratuberculosis. FEMS Microbiol. Lett. 209:267-271.

Ferguson, J. D., D. T. Galligan, and N. Thomsen. 1994. Principal descriptors of body condition score in Holstein cows. J. Dairy Sci. 77:2695-2703.

Hope, A. F., P. F. Kluver, S. L. Jones, and R. J. Condron. 2000. Sensitivity and specificity of two serological tests for the detection of ovine paratuberculosis. Aust. Vet. J. 78:850-856.

Jakobsen, M. B., L. Alban, and S. S. Nielsen. 2000. A cross-sectional study of paratuberculosis in 1155 Danish dairy cows. Prev. Vet. Med. 46:15-27.

Larsen, A. B., R. S. Merkal, and R. C. Cutlip. 1975. Age of cattle as related to resistance to infection with Mycobacterium paratuberculosis. Am. J. Vet. Res. 36:255-257.

Merkal, R. S., D. L. Whipple, J. M. Sacks, and G. R. Snyder. 1987. Prevalence of Mycobacterium paratuberculosis in ileocecal lymph nodes of cattle culled in the United States. JAVMA 190:676-680.

Murray, P. R., ed. 1999. Pages 1678-1679 in Manual of Clinical Microbiology. 7th ed. ASM Press, Washington, DC.

Muskens, J., H. W. Barkema, E. Russchen, K. van Maanen, Y. H. Schukken, and D. Bakker. 2000. Prevalence and regional distribution of paratuberculosis in dairy herds in The Netherlands. Vet. Microbiol. 77:253-261.

Nielsen, S. S., S. M. Thamsborg, H. Houe, and V. Bitsch. 2000. Bulktank milk ELISA antibodies for estimating the prevalence of paratuberculosis in Danish dairy herds. Prev. Vet. Med. 44:1-7.

Ott, S. L., S. J. Wells, and B. A. Wagner. 1999. Herd-level economic losses associated with Johne's disease on US dairy operations. Prev. Vet. Med. 40:179-192.

Pavlik, I., L. Matlova, J. Bartl, P. Svastova, L. Dvorska, and R. H. Whitlock. 2000. Parallel faecal and organ Mycobacterium avium subsp. paratuberculosis culture of different productivity types of cattle. Vet. Microbiol. 77:309-324.
Poupart, P., M. Coene, H. Van Heuverswyn, and C. Cocito. 1993. Preparation of a specific RNA probe for detection of Mycobacterium paratuberculosis and diagnosis of Johne's disease. J. Clin. Microbiol. 31:1601-1605.

Richards, W. D. 1981. Effects of physical and chemical factors on the viability of Mycobacterium paratuberculosis. J. Clin. Microbiol. 14:587-588.

Sergeant, E. S., D. J. Marshall, G. J. Eamens, C. Kearns, and R. J. Whittington. 2003. Evaluation of an absorbed ELISA and an agargel immuno-diffusion test for ovine paratuberculosis in sheep in Australia. Prev. Vet. Med. 61:235-248.

Sockett, D. C. 1996. Johne's disease eradication and control: Regulatory implications. Vet. Clin. North Am. Food Anim. Pract. 12:431-440.

Tiwari, A., J. A. Vanleeuwen, I. R. Dohoo, J. P. Haddad, H. Stryhn, and G. P. Keefe. 2003. Effects of seropositivity for bovine leukosis virus, Mycobacterium avium subspecies paratuberculosis, and Neospora caninum on risk of culling in dairy cattle. Page 160 in Proc. Am. Assoc. Bovine Pract. Columbus, OH.

VanLeeuwen, J. A., G. P. Keefe, R. Tremblay, C. Power, and J. J. Wichtel. 2001. Seroprevalence of infection with Mycobacterium avium subspecies paratuberculosis, bovine leukemia virus, and bovine viral diarrhea virus in maritime Canada dairy cattle. Can. Vet. J. 42:193-198.

Van Schaik, G., C. R. Rossiter, S. M. Stehman, S. J. Shin, and Y. H. Schukken. 2003. Longitudinal study to investigate variation in results of repeated ELISA and culture of fecal samples for Mycobacterium avium subsp. paratuberculosis in commercial dairy herds. Am. J. Vet. Res. 64:479-484.

Vary, P. H., P. R. Andersen, E. Green, J. Hermon-Taylor, and J. J. McFadden. 1990. Use of highly specific DNA probes and the polymerase chain reaction to detect Mycobacterium paratuberculosis in Johne's disease. J. Clin. Microbiol. 28:933-937.

Wells, S. J., and B. A. Wagner. 2000. Herd-level risk factors for infection with Mycobacterium paratuberculosis in US dairies and association between familiarity of the herd manager with the disease or prior diagnosis of the disease in that herd and use of preventive measures. JAVMA 216:1450-1457.

Wells, S. J., R. H. Whitlock, C. J. Lindeman, and T. Fyock. 2002. Evaluation of bacteriologic culture of pooled fecal samples for detection of Mycobacterium paratuberculosis. Am. J. Vet. Res. 63:1207-1211.

Whitlock, R. H. 1996. Preclinical and clinical manifestations of paratuberculosis (including pathology). Vet. Clin. North Am. Food Anim. Pract. 12:345-356.

Whitlock, R. H., S. J. Wells, R. W. Sweeney, and J. Van Tiem. 2000 ELISA and fecal culture for paratuberculosis (Johne's disease): Sensitivity and specificity of each method. Vet. Microbiol. 77:387-398.

Whittington, R. J., and E. S. Sergeant. 2001. Progress towards understanding the spread, detection, and control of Mycobacterium avium subsp. paratuberculosis in animal populations. Aust. Vet. J. 79:267-278. 